\title{
Tactile to Vibrotactile Sensory Feedback Interface for Prosthethic Hand Users
}

\author{
N.H.H. Mohamad Hanif, N. N. Nik Hashim \\ Department of Mechatronics, Kuliyyah of Engineering, \\ International Islamic University Malaysia \\ Kuala Lumpur, Malaysia. \\ noorhazrin@iium.edu.my
}

\begin{abstract}
The motivation of this research work is to provide a sense of embodiment to prosthetic users by supplementing their devices with sensory feedback to the residual upper arm. This sensory feedback replicates the tactile sensory system of glabrous skin that covers palm and flexor surfaces of fingers. In this work, we produced vibration patterns that will be perceived at the upper arm, according to signals obtained by a prosthetic finger when sliding across fabricated textured surfaces. This was done by transforming the signals to 'on' and 'off' pulses in the LabView environment and then forwarded to a data acquisition board to provide voltage signals to a vibration actuator. We implemented a novel frequency measurement procedure to maintain a vibration frequency of $250 \mathrm{~Hz}$, which is the optimum frequency of the mechanoreceptors underneath the skin of the upper arm in detecting vibration. The outcome from this research work leads to optimistic possibility that a touch sensation that was previously lost could be restored to different parts of the body. This undoubtedly will increase users' acceptance of the device as a part of their body due to its 'lifelike' quality.
\end{abstract}

Keywords-prosthetic; embodiment; tactile; vibrotactile; sensory feedback

\section{INTRODUCTION}

The sense of embodiment, or body ownership, makes an important factor for self-recognition. Typically an amputee do not regard the prosthetic device attached to his residual arm as part of his body [1]. Consequently, this becomes one of the factors that the device is under-utilized, and to some extent, rejected regardless the numerous functions offered by the device [2]. However, several research works have shown that the sense of embodiment with prosthetic devices could be improved when sensory feedback is restored [3][4]. Force, pressure and temperature sensors have been designed within the prosthetic device to ensure more control by the user in manipulating the grasped object [5], [6], [7] while a slip sensor made it possible for object slippage prevention [8], [9].

Apart from the capability to manipulate grasped objects, another important feature of a heathy hand that is often neglected is the sense of touch, a sensation exclusively provided by the glabrous skin that covers the palm and fingers. This tactile sensation is very useful as it has superior sensitivity as compared to visual, or even auditory senses [4], [10].

\author{
P.H. Chappell, N.M. White, A.W. Cranny \\ Electronics and Computer Science \\ Faculty of Physical Sciences and Engineering, University of \\ Southampton \\ Southampton, United Kingdom.
}

Raspopovic [11] demonstrated the capability of a bionic hand, wired to a patient's nervous systems that enables him to receive touch signals from the skin sensors. It was reported in works by Di Pino [12] that with the employment of cathodal pulses of $10-300 \mu \mathrm{s}$ with a current intensity between 10-100 $\mu \mathrm{A}$, touch and tingling sensation could be visibly perceived by a transradial amputee. Rager [13] worked with detection of object slip and used the information to reconstruct the force components generated during the slip event to simulate firing responses of skin mechanoreceptors. Another approach in implementing tactile feedback to a prosthetic device was by using ultrathin stretchable silicone nanoribbon electronics as skin prosthesis [14]. The artificial skin was capable to detect strain, humidity, temperature as well as pressure, and passes the sensory signals to peripheral nerves using multi-electrode array. In works by Berg [15], the tactile sensory information was conveyed via intracortical microstimulation of primary somatosensory cortex.

Referring to the list of the published literatures, it could be inferred that most methods in supplementing tactile sensory feedback to the device users involve invasive surgeries. Although proven effective, prospective users may have reservation to this considerably intrusive procedure. Therefore the focus of our research work is to transform the tactile information gathered at the fingertip of the prosthetic device into a vibrotactile sensation that could be felt at the residual upper arm. This stimulation, if not at all painful, could be manipulated to be within the acceptable intensity band to avoid nerve or tissue damages. Also, work by D'Alonzo [16] showed that the vibration supplementary sensation promotes a sense body-ownership of the attached artificial hand in transradial amputees.

In our previous study, we used a piezoelectric sensor attached to a fingertip of a Southampton Hand to acquire vibration signals as textured surface slides past the finger (Fig. 1). With an upgraded data acquisition strategy, the piezoelectric sensor has been able to detect signal frequencies generated during the exploratory movement that reliably correlate to all the widths of grooves and ridges of the surface textures under investigation [17],[18]. 


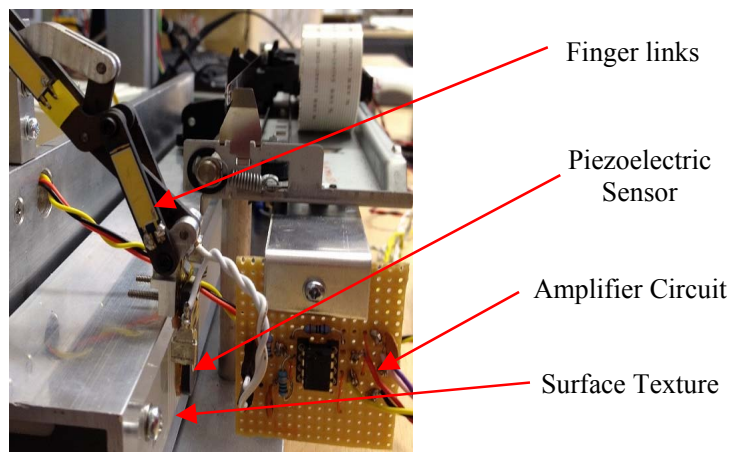

Fig. 1. Data acquisition of the textured surface signals using piezoelectric sensor at the fingertip of the Southampton Hand (reproduced from [18])

We have also investigated the capability of a selected $5 \mathrm{~mm}$ Eccentric Rotation Mass (ERM) motor to become the haptic actuator in producing vibration that works at $250 \mathrm{~Hz}$, which is the optimum frequency of the Pacinian Corpuscle mechanoreceptors located deep underneath the skin of the upper arm [19]. We used the obtained transfer function of the motor to predict its transient responses, and experiments conducted on the motor have provided useful information about the frequency responses and effective vibration energy [20], [21].

In the present work, we concentrated on interfacing the information gathered from the piezoelectric sensor of the artificial hand and the surface texture to create a vibration sensation to be felt on the residual arm. The overall experimental set up for this integration stage is as shown in the following block diagram (Fig. 2). Selected post-processed data from MATLAB that have shown reliable outputs in associating the FFT dominant frequency with the widths of surface gratings were fed into LabView. The 'on' and 'off' pulses were then generated in the LabView environment to represent the surface textures. The signals were later channeled to a data acquisition board, a high voltage isolator and a buffer circuit before becoming input signals to the motor. The motor will rotate according to the 'on' and 'off' signals and produce the associated vibration patterns.

\section{SignAl MANIPULATION}

As reported in our previous work [18], we gathered signals from four surface textures (A, B, C and D) of different dimensions, fabricated on acrylic strips with a length of 100 $\mathrm{mm}$ per texture. Fig. 3 shows the dimensions of the textures.

As the surface textures under investigation were repetition of regular patterns, 'on' and 'off' signal pulses were used to closely represent the textures. 'On' pulses meant that a steady voltage signal was supplied to the vibration motor to mimic the ridge of the surface while the 'off' pulses meant that the voltage signal was cut off (or paused), to represent the groove of the surface texture. The on-off pulses are an effective

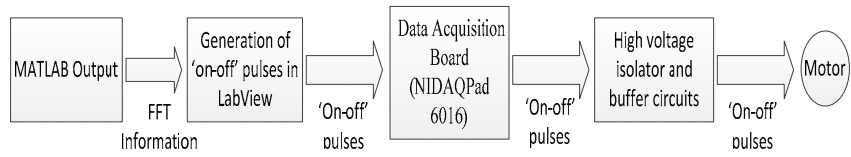

Fig. 2. Block diagram for the interface

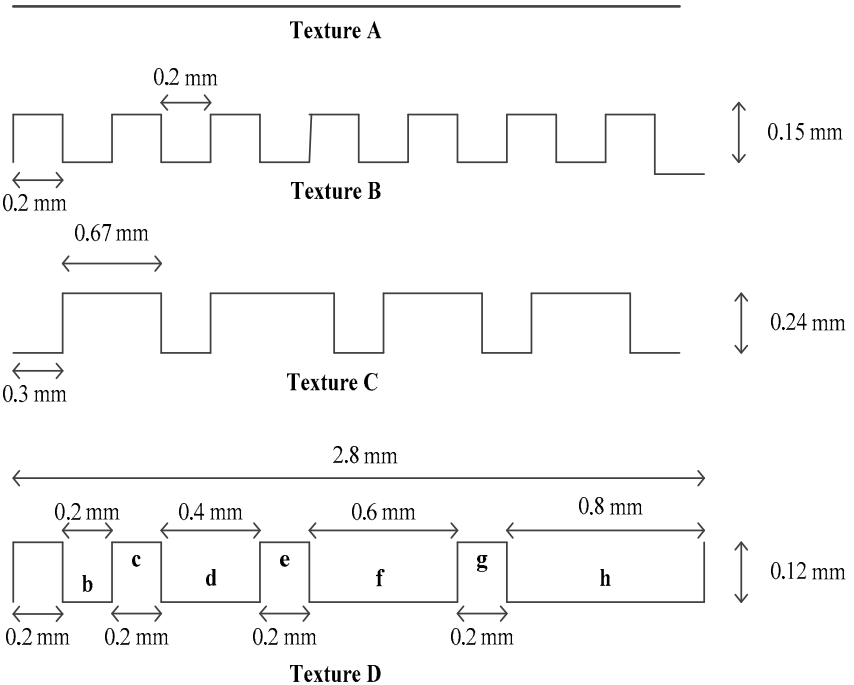

Fig. 3. Dimensions of Surface Textures under Investigation (Texture Asmooth surface, Texture $B$ - repeating every $0.4 \mathrm{~mm}$, Texture $C$ - repeating every $0.97 \mathrm{~mm}$, Texture $D$ - repeating every $2.8 \mathrm{~mm}$ ) (reproduced from [18])

method in perceiving "smooth" or "rough" sensations. A constant speed of vibration is associated to a "smooth" sensation, while short pulses is associated with "rough" sensations [22].

The voltage to be supplied to the haptic device should provide a vibration frequency of $250 \mathrm{~Hz}$ as this is the optimum frequency for mechanoreceptors in the residual arm [19]. From our previous findings, the motor was able to provide a $250 \mathrm{~Hz}$ frequency of vibration when the voltage supplied to the motor was 2V [20]. Therefore input signals were manipulated to provide a constant $2 \mathrm{~V}$ supply for 'on' pulses and $0 \mathrm{~V}$ for 'off' pulses.

The durations of the 'on' and 'off' pulses, T, were calculated by taking the reciprocal of the dominant frequencies, F, obtained using a Fast Fourier Transform (FFT) analysis, which have been verified to estimate the width of grooves and ridges of the surface texture [18]. The calculated values of the duration were scaled according to the transient response of the motor. The duration should be within the capability of the motor as a very short duration will not allow ample time for the motor to be fully in rotation or in braking mode. The durations of the 'on' and 'off' pulses should also not be too long as it may cause discomfort to the users. As reported by Kaaresoja [23], stimuli longer than 200 milliseconds is considered as annoying.

The approximate duration for the 'on' and 'off' pulses to represent the widths of grooves and ridges of the surface textures are as tabulated in Table 1.

\section{Configuration With LABVIEW AND DATA ACQUiSition BOARD}

The durations, sequence and the number of cycles of the 'on' and 'off' pulses for all four surface textures were programmed in LabView. For ease of psychophysical investigation at the later stage, the LabView programs for all four surface textures were done in separate block diagrams. 
TABLE I. DESCRIPTIONS OF THE VIBRATION PATTERNS AND THEIR ASSOCIATED SURFACE TEXTURES

\begin{tabular}{|c|l|c|}
\hline $\begin{array}{c}\text { Vibration } \\
\text { Pattern }\end{array}$ & \multicolumn{1}{|c|}{ Descriptions } & $\begin{array}{c}\text { Associated } \\
\text { Texture }\end{array}$ \\
\hline Pattern A & $\begin{array}{l}\text { Continuous vibration (2000 } \\
\text { millisecond) }\end{array}$ & Texture A \\
\hline Pattern B & $\begin{array}{l}\text { Repeated 500 millisecond* pulses } \\
\text { with 500 millisecond* pauses in } \\
\text { between }\end{array}$ & Texture B \\
\hline Pattern C & $\begin{array}{l}\text { Repeated 1000 millisecond* pulses } \\
\text { with 500 millisecond* pauses in } \\
\text { between }\end{array}$ & $\begin{array}{l}\text { Texture C } \\
\text { Pepeated 250 millisecond* pulses } \\
\text { Pith 250 millisecond* pause in } \\
\text { between, then 250 millisecond* pulses } \\
\text { with 500 millisecond pause in } \\
\text { between, then 250 millisecond* pulses } \\
\text { with 750 millisecond* pauses in } \\
\text { between, and finally 250 millisecond* } \\
\text { pulses with } 1000 \text { millisecond* pauses } \\
\text { in between }\end{array}$ \\
\hline
\end{tabular}

As two states ('on' and 'off') were involved, we utilized the state machine virtual instrument in this program. The duration for the 'on' and 'off' states were dependent on the dominant frequencies while the repetitions of the vibration patterns were implemented using a counter. The number of cycles was user defined and could be controlled at the front panel of the LabView.

We utilized the NIDAQPad-6016, a data acquisition device to channel out the pulses to the motor in generating the associated vibration patterns. In interfacing the software (programming) and the hardware (motor circuitry), a virtual channel had to be defined in the LabView environment. The virtual channel specified the output terminals that have been wired. We opted for the DAQmx virtual instruments to ensure a more flexible control of the output.

\section{HARDWARE CONFIGURATION}

\section{A. Electronic Circuitries}

The output pulses from LabView became the input signals to the motor circuitry in generating vibration output. A circuit, shown in Fig. 4 was specifically built to amplify the current supply, protect the data acquisition board and isolate the motor from the mains supply. The input pulses from the DAQ will be fed into the isolator circuit. The output from the isolator was connected to the input of an inverter and then to the $22 \mathrm{k} \Omega$ potentiometer of the buffer circuit which will in turn power the motor.

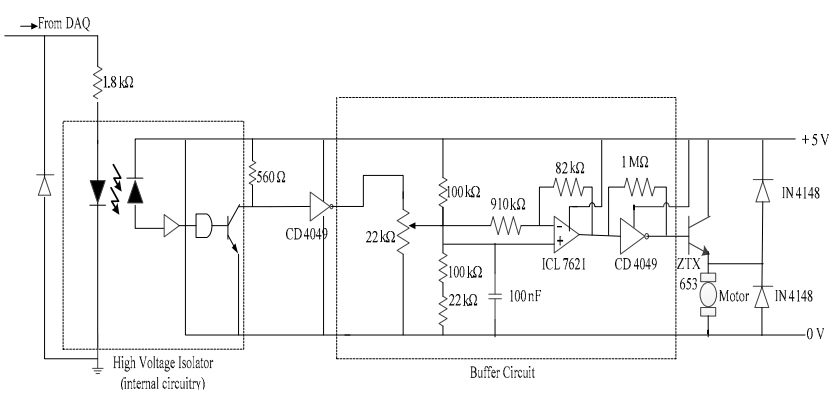

Fig. 4. Connection between the DAQ, isolator and the buffer circuit
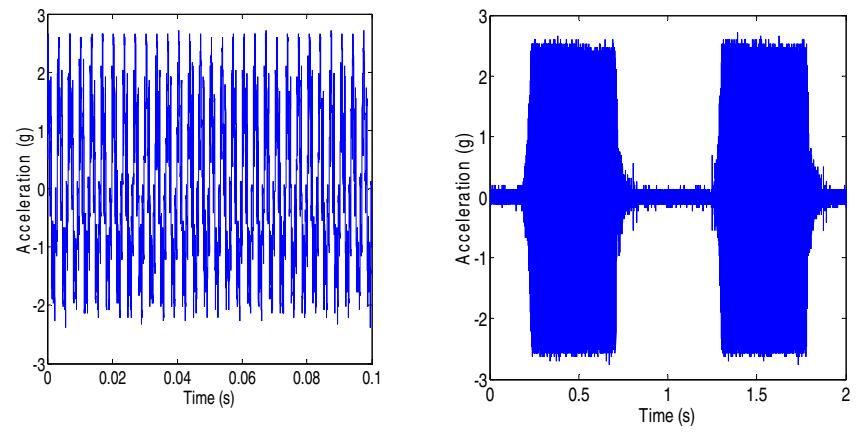

Pattern A (continuous vibration)

Pattern B

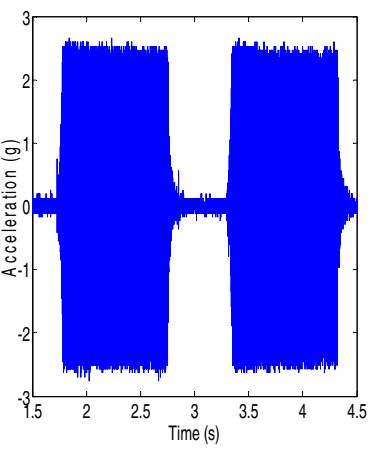

Pattern C

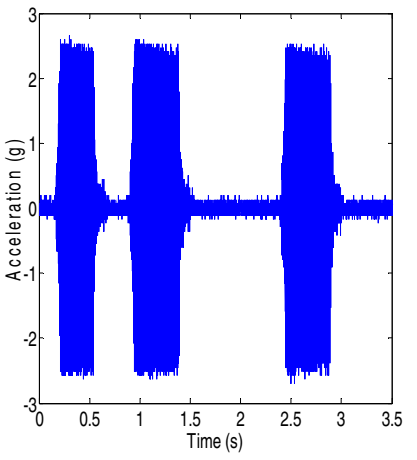

Pattern D

Fig. 5. Acceleration in $x$-axis direction for vibration patterns $A, B, C$ and D

\section{B. Output signals}

The input signals from the DAQ board were tested to see its capability in producing similar signals as programmed in the LabView environment. The signals were the four vibration patterns designed according to the acquired information of the surface textures. We mounted the motor onto an accelerometer, and measured the acceleration $(\mathrm{g})$ and dominant frequencies for all four vibration patterns in the $\mathrm{x}$-axis direction. The output plots for all four patterns are shown in Fig. 5.

A further analysis was conducted to check the transient responses of the motor when subjected to the 'on' and 'off' pulses. The average transient responses for the generated vibration patterns were 41.5 and 35.5 milliseconds for rise time and stop time respectively. These transient responses, due to the starting and braking of the motor have been anticipated. Therefore to ensure sufficient vibration could be perceived at the upper arm, 80 milliseconds were added for every duration of the 'on' and 'off' pulses to counter the time losses during the state transitions.

\section{Frequency Measurement Procedure}

To ensure the vibration patterns could be effectively perceived, the frequency of vibration of the motor should be $250 \mathrm{~Hz}$. This is the peak frequency for the characteristics of the mechanoreceptors underneath the skin of the upper arm in detecting vibration [19].

We observed however, that the frequency of vibration of the output signals tended to vary although the input signal to 
the motor was constant. This variation was due to how the motor was mounted. When the motor was held stationary on top of the table, the desired $250 \pm 10 \mathrm{~Hz}$ frequency of vibration could be achieved. However when the motor was held loosely on top of different surfaces, the frequency varied greatly. As the motor will be later attached on the upper arm, there will be high possibilities that the frequency of vibration may not be within the desired range. To address this situation, we implemented a frequency measurement and control method to confirm and maintain the frequency.

The vibration frequencies were determined through FFT analysis of the motor terminal voltage. The ERM motor consisted of three poles; hence the commutation points were three times for each revolution. To measure the frequency of vibration per revolution, the positive and negative terminals of the motor were connected to the probes of a digital oscilloscope. The motor was supplied with voltage from the DAQ board through the buffer and isolator circuits, and this input voltage was observed at the oscilloscope Fig. 6 (a)).

We utilized the FFT function of the oscilloscope to obtain the frequency content for one revolution of the motor. As shown in Fig. 6(b), this was done by focusing the FFT calculation at the $100 \mathrm{mV}$ ac of the input signal, which was the periodic variation produced by the rotation of the motor. To ensure only vibration from the rotating mass was measured, the body of the motor was held stationary on top of a table during the measurement using a finger.

In one revolution, the motor should ideally produce $250 \mathrm{~Hz}$ of vibration frequency. From Fig. 6(b), the period (T) for one revolution of the motor is 3.5 milliseconds. Therefore, the frequency for one motor revolution is $1 / \mathrm{T}=285.7 \mathrm{~Hz}$. Thus the input voltage to the motor needed to be further adjusted to achieve to the desired frequency. As the oscilloscope provided a real time measurement from the motor, the dominant frequency could be monitored and controlled. The dominant frequency could be easily changed by adjusting the potentiometer of the buffer circuit. An FFT plot from an analysis of the motor voltage is shown in Fig. 7.

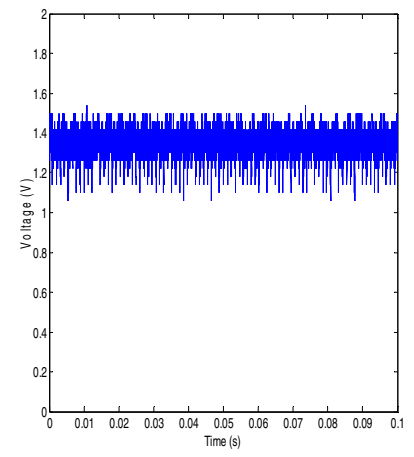

(a)

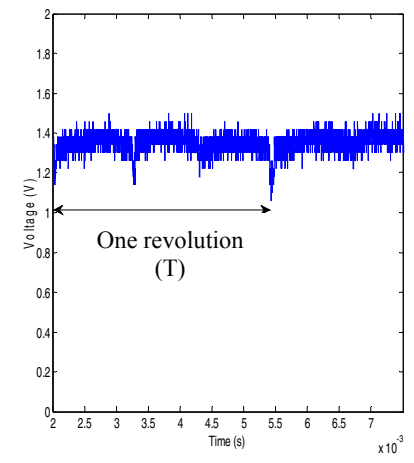

(b)
Fig. 6. Input voltage to the motor (left) and a zoomed in figure of the same input voltage to highlight one revolution of the motor (right)

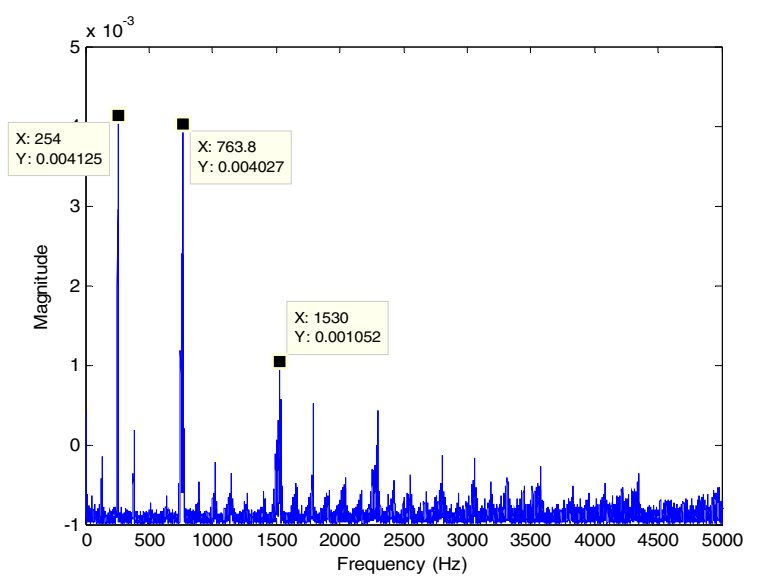

Fig. 7. Direct FFT measurement of the vibration motor

The plot in Fig. 7 showed dominant frequencies at $254 \mathrm{~Hz}$, $763.8 \mathrm{~Hz}$ and $1530 \mathrm{~Hz}$, with $254 \mathrm{~Hz}$, within the desired range of frequency, being the most dominant. The subsequent dominant frequencies were multiples of 3 of the first dominant frequency. This was a result of the construction of the motor which consisted of three poles. The multiples of 3 of the dominant frequencies $(3 \times 245 \mathrm{~Hz}=762 \mathrm{~Hz}, 6 \times 254 \mathrm{~Hz}=$ $1524 \mathrm{~Hz}$ ) confirmed that the motor was working accordingly and that the frequency could be manipulated according to the desired values.

\section{DISCUSSIONS}

In this work, we generated the vibration patterns from the saved data previously gathered by the piezoelectric sensor from the textured surfaces. The selected data has been postprocessed in MATLAB and had shown repeatable accurate outputs in relating the dominant frequency of FFT analysis with the widths of the texture gratings. This provides great confidence that the textures have been reliably represented by the FFT output, and by carrying forward the obtained information, the likelihood that the generated vibration patterns to represent the surface textures is high.

It is also a good practice to always check the frequency of operation of the motor. Although the voltage input provided to the vibration motor is the same throughout the investigation, the vibration output may greatly vary. A soft surface will result in lower frequency of vibration as it absorbs more vibration as compared to the hard surface. On top of that, the working condition of the motor may also deteriorate over time. Additionally, the inertia of the motor mounting platform will also affect the frequency of vibration.

Thus by implementing the frequency measurement procedure, not only the frequency could be monitored and instantly adjusted to the desired value, the condition of the motor could also be verified from the FFT output. This is because the dominant frequencies could be determined according to the number of poles of the motor. Apart from its effectiveness, this procedure only requires a low cost ERM motor to provide the associated vibration sensation due to its physical characteristics. 


\section{CONCLUSION AND FUtURE RESEARCH}

The integration of tactile and haptic premises for prosthetic hands has been successfully demonstrated in this research work. The haptic actuator, which is the eccentric rotation mass (ERM) motor, was able to produce associated vibration patterns with reference to the applied input pulses at a total delay time of less than 90 milliseconds. The implementation of the frequency measurement technique has improved the accuracy of the vibration actuator to produce a vibration frequency within a narrow bandwidth of $250 \mathrm{~Hz}$, the optimum frequency in detecting vibration at the upper arm. This developed technique ensures consistent setting of the vibration stimuli on the upper arm and is therefore not a factor to be considered having an influence during testing human responses.

The results from this research have shown promising implementation of sensory feedback to upper prosthetic device users. The next stage of this research work will be psychophysics evaluation on healthy participants to see whether both tactile and vibrotactile sensations could be discriminated and matched accordingly.

\section{REFERENCES}

[1] F. De Vignemont, "Embodiment, ownership and disownership," Conscious. Cogn., vol. 20, no. 1, pp. 82-93, 2011.

[2] E. A. Biddiss and T. T. Chau, "Upper limb prosthesis use and abandonment: a survey of the last 25 years.," Prosthet. Orthot. Int., vol. 31, no. 3, pp. 236-57, Sep. 2007.

[3] J. Gonzalez, H. Soma, M. Sekine, and W. Yu, "Psycho-physiological assessment of a prosthetic hand sensory feedback system based on an auditory display: a preliminary study.,"J. Neuroeng. Rehabil., vol. 9, p. 33, Jan. 2012

[4] Y. Visell, "Tactile sensory substitution: Models for enaction in HCI," Interact. Comput., vol. 21, no. 1-2, pp. 38-53, Jan. 2009.

[5] A. Cranny, D. P. J. Cotton, P. H. Chappell, S. P. Beeby, and N. M. White, "Thick-film force, slip and temperature sensors for a prosthetic hand," Meas. Sci. Technol., vol. 16, no. 4, pp. 931-941, Apr. 2005.

[6] A. Chatterjee, P. Chaubey, J. Martin, and N. Thakor, "Testing a Prosthetic Haptic Feedback Simulator With an Interactive Force Matching Task," JPO J. Prosthetics Orthot., vol. 20, no. 2, pp. 27-34, Apr. 2008.

[7] K. Kim and J. Colgate, "On the design of miniature haptic devices for upper extremity prosthetics," IEEE/ASME Trans. Mechatronics, vol. 15, no. 1, pp. 27-39, 2010.

[8] D. Cotton, P. Chappell, and A. Cranny, "A novel thick-film piezoelectric slip sensor for a prosthetic hand," IEEE Sens. J., vol. 7, no. 5, pp. 752761, 2007.

[9] L. Beccai, S. Roccella, L. Ascari, P. Valdastri, A. Sieber, M. C.
Carrozza, A. Member, and P. Dario, "Development and Experimental Analysis of a Soft Compliant Tactile Microsensor for Anthropomorphic Artificial Hand," vol. 13, no. 2, pp. 158-168, 2008.

[10] T. Mano and M. Ohka, "Mechanisms of fine-surface-texture discrimination in human," J. Accoust. Soc. Am., vol. 105, no. 4, pp. 2485-2492, 1999.

[11] S. Raspopovic and M. Capogrosso, "Restoring Natural Sensory Feedback in Real-Time Bidirectional Hand Prostheses," Sci. Transl. Med., vol. 6, no. 222, 2014.

[12] G. Di Pino and A. Benvenuto, "Implant of Intraneural Multielectrodes in Human for Controlling a 5-fingered Hand Prosthesis, delivering sensorial feedback and producing rehabilitative neuroplasticity," in The Fourth IEEE RAS/EMBS International Conference on Biomedical Robotics and Biomechatronics, 2012, pp. 1831-1836.

[13] D. M. Rager, D. Alvares, I. Birznieks, S. J. Redmond, J. W. Morley, N. H. Lovell, and R. M. Vickery, "Generating tactile afferent stimulation patterns for slip and touch feedback in neural prosthetics," Proc. Annu. Int. Conf. IEEE Eng. Med. Biol. Soc. EMBS, pp. 5922-5925, 2013.

[14] J. Kim, M. Lee, H. J. Shim, R. Ghaffari, H. R. Cho, D. Son, Y. H. Jung, M. Soh, C. Choi, S. Jung, K. Chu, D. Jeon, S.-T. Lee, J. H. Kim, S. H. Choi, T. Hyeon, and D.-H. Kim, "Stretchable silicon nanoribbon electronics for skin prosthesis.," Nat. Commun., vol. 5, pp. 1-11, 2014.

[15] J. A. Berg, F. V Tenore, L. Jessica, R. J. Vogelstein, and S. J. Bensmaia, "Restoring the sense of touch with a prosthetic hand through a brain interface," Proc. Natl. Acad. Sci., vol. 111, no. 2, pp. 18279-18284, 2014.

[16] M. D’Alonzo, F. Clemente, and C. Cipriani, "Vibrotactile stimulation promotes embodiment of an alien hand in amputees with phantom sensations.," IEEE Trans. Neural Syst. Rehabil. Eng., vol. 23, no. 3, pp. 450-457, 2014.

[17] P. H. Chappell, N. Muridan, A. Mohamad Hanif, N. Hazrin H., Cranny, and N. M. White, "Sensing texture using an artificial finger and a data analysis based on the standard deviation," IET Sci. Meas. Technol., pp. 1-9, 2015.

[18] N. H. H. M. Hanif, P. H. Chappell, A. Cranny, and N. M. White, "Surface Texture Detection with Artificial Fingers," in 37th Annual International Conference of IEEE Engineering in Medicine and Biology Society, 2015, pp. 8018-8021.

[19] E. Kandel, J. Schwartz, and T. Jessell, Principles of neural science, 4th ed., no. 1. McGraw Hill, 2000.

[20] N. H. H. M. Hanif, P. H. Chappell, A. Cranny, and N. M. White, "Development of Vibrotactile Sensory Feedback For Prosthetic Hand Users," in IASTED International Conference Biomedical Engineering (BioMed 2014), 2014.

[21] N. H. H. M. Hanif, P. H. Chappell, A. Cranny, and N. M. White, "Vibratory feedback for artificial hands," in 2013 International Conference on Electronics, Computer and Computation (ICECCO), 2013, pp. 247-250.

[22] L. Brown and T. Kaaresoja, "Feel who's talking: using tactons for mobile phone alerts," CHI'06 Ext. Abstr. Hum. factors Comput. Syst., pp. 604-609, 2006.

[23] T. Kaaresoja and J. Linjama, "Perception of Short Tactile Pulses Generated by a Vibration Motor in a Mobile Phone," First Jt. Eurohaptics Conf. Symp. Haptic Interfaces Virtual Environ. Teleoperator Syst., pp. 471-472, 2005. 